\title{
Is Phenalenyl Aromatic?
}

\author{
Jerry Ray Dias
}

\author{
Department of Chemistry, University of Missouri, Kansas City, MO 64110-2499 \\ Corresponding author's e-mail address: diasj@umkc.edu
}

RECEIVED: November 5, 2019 * REVISED: December 27, 2019 * ACCEPTED: December 28, 2019

PROCEEDING OF THE 31ST MATH/CHEM/COMP CONFERENCE, JUNE 10-14, 2019, DUBROVNIK, CROATIA DEDICATED TO THE MEMORY OF DR. EDWARD CAMERON KIRBY, FRIEND AND COLLEAGUE

Abstract: The rings phenalenyl are determined using some quantitative aromatic indices to have around $40 \%$ the aromaticity of benzene. The monocation, monoanion, and monoradical of phenalenyl are found to be equally aromatic.

Keywords: aromaticity, topological resonance energy (TRE), bond resonance energy (BRE), energy effect (ef), conjugated circuit energy, HOMA.

\section{INTRODUCTION}

$\mathbf{M}$ OST indices for measuring aromaticity have been developed and demonstrated for nonradical benzenoid hydrocarbons which include Aihara's topological resonance energy (TRE) for global aromaticity and bond resonance energy for local ring aromaticity. ${ }^{[1]}$ Harmonic Oscillator Model of Aromaticity (HOMA), ${ }^{[2]}$ energy effect (circuit resonance energy (CRE) or ef)., ${ }^{[3,4]}$ and conjugated circuit theory. ${ }^{[5]}$ Of these various indices, radical benzenoid hydrocarbons have been largely overlooked. We will frequently use the term benzenoid for benzenoid hydrocarbon. While Randić in his extensive review of aromaticity of polycyclic conjugated hydrocarbons excluded radical polycyclic systems in his definition benzenoid hydrocarbons (page 3516), we will include them as being benzenoid.. ${ }^{[6]}$ Here we explore the use of these indices for measuring degree of aromaticity of global and local ring aromaticity of phenalenyl monoradical $\left(\mathrm{C}_{13} \mathrm{H}_{9}\right)$. Phenalenyl is the smallest radical benzenoid hydrocarbon. All odd carbon benzenoids are radical conjugated systems with an antiaromatic perimeter like phenalenyl monoradical which has a C12 circuit shown in Figure 1. The second smallest monoradical benzenoid is benzo[cd]pyrene $\left(\mathrm{C}_{19} \mathrm{H}_{11}\right)$ which has an antiaromatic $\mathrm{C} 16$ perimeter circuit. For comparison the benzene with a C6 circuit and [10]-, [12]-, and [16]-annulenes have TRE of $0.2726,0.1594,-0.3944$, and $-0.2952 \beta$, respectively. This shows two things. First, antiaromaticity is stronger than aromaticity. Second, both antiaromaticity and aromaticity decrease with increasing circuit size.

The electron spin resonance spectrum (ESR) of phenalenyl monoradical itself was reported in $1957^{[7]}$ and its 2,5,8-tri-tert-butyl-phenalenyl derivative was synthesized and fully characterized. ${ }^{[8]}$ It is of interest that nucleusindependent chemical shift (NICS) analysis of 2,5,8-tri-tertbutyl-phenalenyl showed that the ring center of the dimer became more aromatic $(-7.1 \mathrm{ppm})$ than that of the monomer $(-3.8 \mathrm{ppm}) .{ }^{[9]}$ The issue of aromaticity of phenalenyl motif was the subject of a paper which focused on perimeter ring current density patterns. ${ }^{[10]}$

We will first examine the global aromaticity/antiaromaticity and resonance of phenalenyl monoradical and then evaluate the aromaticity of phenalenyl monoradical with the quantitative aromatic indices of Aihara (TRE and BRE), Bosanac and Gutman (ef), Krygowski (HOMA), and Randić's (partitioned conjugated circuit theory).

\section{RESULTS AND DISCUSSION}

\section{Topological Resonance Energy (TRE) and Global Aromaticity of Phenalenyl}

Topological resonance energy (TRE) is a measure of the excessive stabilization energy due to cyclic conjugation and ring current electron delocalization associated with aromaticity. It is defined within the Hückel molecular orbital 
(HMO) framework. ${ }^{[1]}$ The sum of the positive roots multiplied by their occupation numbers of the characteristic polynomial of a polycyclic aromatic hydrocarbon (PAH) gives its total pr-electronic energy $\left(E_{\pi}\right)$ and the sum of the positive roots multiplied by their occupation numbers of the matching polynomial gives the pr-electronic energy $E_{\pi}($ ref) to the hypothetical reference polyene to original $\mathrm{PAH}$. The characteristic and matching polynomials were computed using the Balasubramanian software programs. ${ }^{[11,12]}$ The matching polynomial excludes cyclic Sachs graph contributions while maintaining the same conjugation. The difference in these two pr-electronic energies gives the TRE $=E_{\pi}-E_{\pi}($ ref $)$ of the PAH. This energy difference represents the extra stabilization energy due to aromatic stabilization energy (ASE). The hypothetical reference polyene has the same geometry, the same number of $\pi$-electrons, the same electronic configuration, the same spin multiplicity, the same conjugation energy, and the same steric strain. Aihara has shown that the TRE concept can be extended to charged $\pi$-systems. \%TRE is defined as TRE divided by the total $\pi$ binding energy of the polyene reference structure $\left[E_{\pi}(\right.$ ref $\left.)\right]$ and multiplied by 100 . This normalizes TRE with respect to the size of the $\pi$-system and can be used to compare the aromaticity of organic molecules of different sizes.

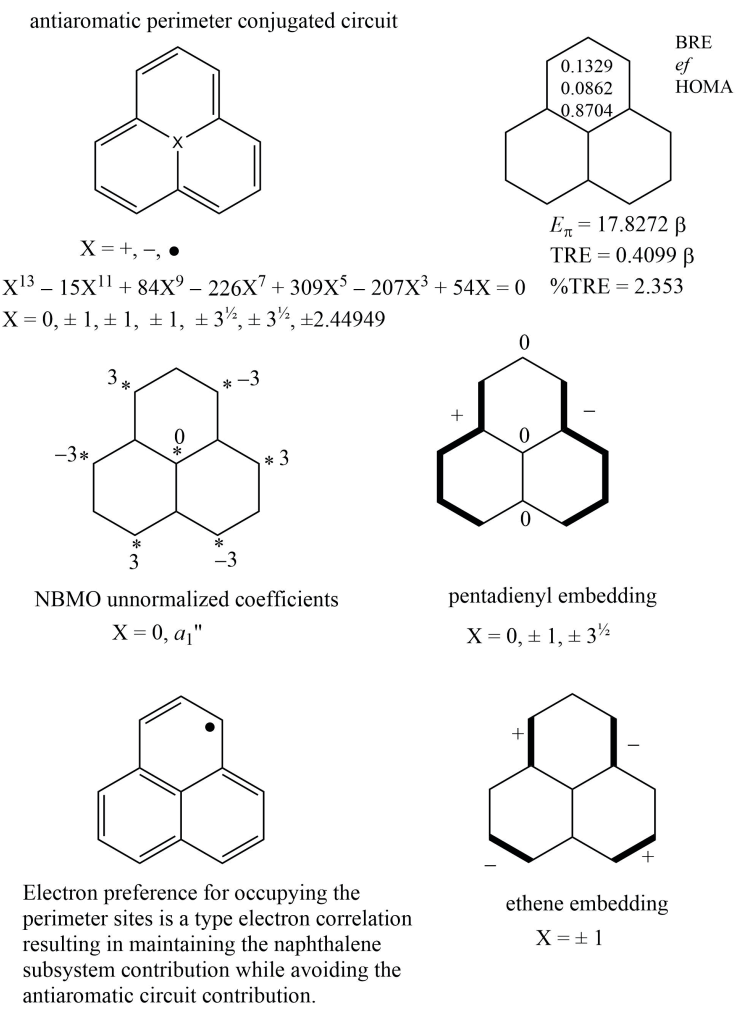

Figure 1. Analysis of $\mathrm{HMO}$ electronic characteristics phenalenyl.
Open-shell species have far more resonance structures than their closed-shell isomers. We agree with Aihara that open-shell species can be kinetically very reactive but still acquire some aromatic stabilization. ${ }^{[1]}$ The HMO double degeneracy in Figure 2 is in agreement with higher level calculations. ${ }^{[13]}$ Figure 2 summarizes the TRE calculations of for phenalenyl. What is amazing is that both the monoanion and monocation species are both determined to be aromatic with the same TRE $=0.40984 \beta$. This result was also obtained by Cyranski and coworkers using higher level calculations and $\pi$-current-density maps. ${ }^{[10]}$ Our valencebond (VB) interpretation follows. The electron or charge on phenalenyl is solely delocalized on the perimeter because the eigenvector coefficient of the nonbonding molecular orbital (NBO) at the phenalenyl central position is zero (Figure 1). Calculations using $\pi-M R-A Q C C / C A S(7,7) / 6311 G(2 d)$ for the $A_{1}$ " ground state shows that the central carbon atom of phenalenyl does not carry any unpaired electron density. ${ }^{[13]}$ This results in avoidance of the participation of the antiaromatic perimeter $\mathrm{C} 12$ circuit while maintaining the naphthalene aromatic electronic subsystem in all of the 18 resonance structures. This is a type of VB electron correlation. Naphthalene has \%TRE $=2.924$ and pentacene has \%TRE $=2.105$ compared to phenalenyl which has \%TRE =<smiles>C[C@H]1CCC2CCCC3CCC[C@H](C2)C31</smiles>

characteristic polynomial and eigenvalues $\mathrm{X}^{13}-15 \mathrm{X}^{11}+84 \mathrm{X}^{9}-226 \mathrm{X}^{7}+309 \mathrm{X}^{5}-207 \mathrm{X}^{3}+54 \mathrm{X}=0$

$\mathrm{X}=0, \pm 1, \pm 1, \pm 1, \pm 3^{1 / 2}, \pm 3^{1 / 2}, \pm 2.44949$

trianion/diradical $E_{\pi}=15.82718 \beta$

monoanion $E_{\pi}=17.82718 \beta$

monoradical $E_{\pi}=17.82718 \beta$

monocation $E_{\pi}=17.82718 \beta$

trication/diradical $E_{\pi}=15.82718 \beta$

matching polynomial and eigenvalues

$\mathrm{X}^{13}-15 \mathrm{X}^{11}+84 \mathrm{X}^{9}-220 \mathrm{X}^{7}+273 \mathrm{X}^{5}-141 \mathrm{X}^{3}+20 \mathrm{X}=0$

$X=0, \pm 0.46990, \pm 0.92684, \pm 1.34352, \pm 1.68493, \pm 1.91587, \pm 2.36760$

trianion $E_{\pi}(\mathrm{ref})=16.47754 \beta$

monoanion $E_{\pi}(\mathrm{ref})=17.41734 \beta$

monoradical $E_{\pi}(\mathrm{ref})=17.41734 \beta$

monocation $E_{\pi}(\mathrm{ref})=17.41734 \beta$

trication $E_{\pi}(\mathrm{ref})=16.47754 \beta$

$\operatorname{TRE}($ trianion $)=E_{\pi}-E_{\pi}(\mathrm{ref})=15.82718 \beta-16.47754 \beta=-0.65036 \beta$ $\operatorname{TRE}($ monoanion $)=E_{\pi}-E_{\pi}(\mathrm{ref})=17.82718 \beta-17.41734 \beta=0.40984 \beta$ $\operatorname{TRE}($ monoradical $)=E_{\pi}-E_{\pi}(\mathrm{ref})=17.82718 \beta-17.41734 \beta=0.40984 \beta$ $\operatorname{TRE}($ monocation $)=E_{\pi}-E_{\pi}(\mathrm{ref})=17.82718 \beta-17.41734 \beta=0.40984 \beta$ $\operatorname{TRE}($ trication $)=E_{\pi}-E_{\pi}(\mathrm{ref})=15.82718 \beta-16.47754 \beta=-0.65036 \beta$

Figure 2. Calculation of topological (global) resonance energy (TRE) of phenalenyl. 
2.353. Thus, by this measure both the singly charged and radical phenalenyl have a moderate global aromaticity even though they may be kinetically reactive.

By VB the trication and trianion of phenyleneyl are closed-shell antiaromatic species but by $\mathrm{HMO}$ calculations they are open-shell antiaromatic diradicals (Figure 2) as a result of symmetry induced degeneracy. ${ }^{[14]}$

\section{Local Ring Aromaticity}

Hexagonal ring aromaticity can be determined by $B R E$, ef, HOMA, and partitioned conjugated circuit theory. Aihara defined BRE as the HMO standard resonance integral $(\beta)$ between two adjacent carbon $p \pi$ orbitals $C_{p}-C_{q} \pi$-bond be assigned $\beta_{\mathrm{pq}}=i \beta$ and $\beta_{\mathrm{qp}}=-i \beta$ where $i$ is the square root of -1 for a hypothetical $\pi$-system. By this cyclic conjugation through this $\pi$-bond is blocked. In this hypothetical $\pi$-system, no circulation of $\pi$-electrons is expected along the circuits sharing the $C_{p}-C_{q}$ bond in common. ${ }^{[1]}$ The BRE for this selected $C_{p}-C_{q}$ bond is the destabilization energy of this hypothetical $\pi$-system relative to the actual one. We use the following alternative graph-theoretical method ${ }^{[15]}$ to determine the characteristic polynomial $\left[P\left(G_{\mathrm{ref}} ; \mathrm{X}\right)\right]$ of this hypothetical reference $\pi$-system $\left(G_{\text {ref }}\right)$ associated with a given actual system $(G)$ which circumvents using a determinant as done by Aihara:

$$
P\left(G_{\text {ref }} ; \mathrm{X}\right)=P(G-e ; \mathrm{X})-P(G-(e) ; \mathrm{X})
$$

where $G-e$ is the molecular graph of $G$ with edge $C_{p}-C_{q}$ deleted and $G-(e)$ is the molecular graph of $G$ with edge $C_{p}-C_{q}$ and its corresponding vertices deleted. BRE for a given peripheral $\pi$-bond represents the removal of all the circuits in original molecule from the hypothetical reference molecule that enclose the target ring. All circuits that do not enclose the target ring remain in the hypothetical reference molecule. The $\pi$-bonding energy $E_{\pi}($ ref) of the hypothetical reference molecule is subtracted from the $\pi$-bonding energy $E_{\pi}$ of the original molecule, i.e., $\mathrm{BRE}=$ $E_{\pi}-E_{\pi}($ ref). This process removes the stabilizing and/or destabilizing circuit energies associated with the target ring. While BRE for any peripheral $\pi$-bond stems from all circuits that pass through the bond, the length-six circuit must make the largest contribution. This is why BRE for any peripheral $\pi$-bond can be used as a local aromaticity index. ${ }^{[16]}$

$B R E=0.1329 \beta$ for the any of the three equivalent peripheral hexagonal rings of phenalenyl (Figure 3 ) which is about half ( $49 \%$ ) the BRE $=0.2726 \beta$ for benzene. This value has excluded $\mathrm{C} 6,2 \mathrm{C} 10$, and $\mathrm{C} 12$ circuits but retained $2 \mathrm{C} 6$ and $\mathrm{C} 10$ in the reference molecule. This BRE $=0.133 \beta$ for the three equivalent rings of phenalenyl can be compared to $\mathrm{BRE}=0.210 \beta$ the two equivalent rings of naphthalene. Three times the ring BRE values of $0.133 \beta$ gives $0.399 \beta$ which nearly equals the global TRE $=0.410 \beta$ for phenalenyl, and two times the ring BRE values of $0.210 \beta$ gives $0.420 \beta$ which nearly equals the global TRE $=0.389 \beta$ for naphthalene.

In 1977 Bosanac and Gutman ${ }^{[3]}$ proposed a measure of circuit resonance energy for polycyclic $\pi$-conjugated systems which is closely related to Aihara's BRE when applied to hexagonal rings. The difference between the two different measures is the hypothetical reference system used. BRE includes all circuits passing through the selected bond where the smallest hexagonal circuit is the most important one because aromaticity rapidly diminishes with increasing circuit size. The characteristic polynomial $\left[P\left(G-c_{i} ; \mathrm{X}\right)\right]$ of the Bosanac and Gutman hypothetical reference system $G-c_{i}$ is graph $G$ with the ith cicuit deleted. It is determined as follows. Here $G-c_{i}$ has the same topology as $G$ but is assumed to have no ith circuit when applying the Sachs theorem. The characteristic polynomial of $G-c_{i}$ is determined by

$$
P\left(G-c_{i} ; \mathrm{X}\right)=P(G ; \mathrm{X})+2 P\left(G-r_{i} ; \mathrm{X}\right)=P\left(G_{\text {ref }} ; \mathrm{X}\right)
$$

where $G-r_{i}$ is the molecular graph of $G$ in which the ith ring

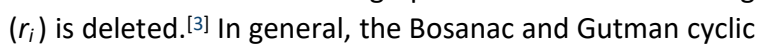
conjugation energy (CCE) is given by

$$
\mathrm{CCE}=e f=E(G)_{\pi}-E\left(G_{\mathrm{ref}}\right)_{\pi}
$$

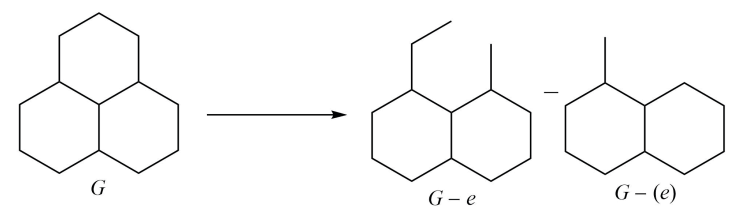

characteristic polynomial and eigenvalues

$\mathrm{X}^{13}-15 \mathrm{X}^{11}+84 \mathrm{X}^{9}-226 \mathrm{X}^{7}+309 \mathrm{X}^{5}-207 \mathrm{X}^{3}+54 \mathrm{X}=0$

$\mathrm{X}=0, \pm 1, \pm 1, \pm 1, \pm 3^{1 / 2}, \pm 3^{1 / 2}, \pm 2.44949$

trianion/diradical $E_{\pi}=15.82718 \beta$

monoanion $E_{\pi}=17.82718 \beta$

monoradical $E_{\pi}=17.82718 \beta$

monocation $E_{\pi}=17.82718 \beta$

trication/diradical $E_{\pi}=15.82718 \beta$

BRE reference polynomial and eigenvalues

$P\left(G_{\mathrm{ref}} ; \mathrm{X}\right)=P(G-e ; \mathrm{X})-P(G-(e) ; \mathrm{X})$

$\mathrm{X}^{13}-14 \mathrm{X}^{11}+72 \mathrm{X}^{9}-174 \mathrm{X}^{7}+206 \mathrm{X}^{5}-111 \mathrm{X}^{3}+20 \mathrm{X}$

$-\left(X^{11}-12 X^{9}+50 X^{7}-91 X^{5}+72 X^{3}-20 X\right)$

$\mathrm{X}^{13}-15 \mathrm{X}^{11}+84 \mathrm{X}^{9}-224 \mathrm{X}^{7}+297 \mathrm{X}^{5}-183 \mathrm{X}^{3}+40 \mathrm{X}=0$

$X=0, \pm 0.68935, \pm 1.0, \pm 1.26560, \pm 1.60589, \pm 1.86217, \pm 2.42415$

trianion/diradical $E_{\pi}(\mathrm{ref})=16.31561 \beta$

monoanion $E_{\mathrm{T}}(\mathrm{ref})=17.69431 \beta$

monoradical $E_{\pi}(\mathrm{ref})=17.69431 \beta$

monocation $E_{\pi}(\mathrm{ref})=17.69431 \beta$

trication/diradical $E_{\pi}(\mathrm{ref})=16.31561 \beta$

$\mathrm{BRE}($ trianion $)=E_{\pi}-E_{\pi}(\mathrm{ref})=15.82718 \beta-16.31561 \beta=-0.48843 \beta$ $\mathrm{BRE}($ monoanion $)=E_{\alpha}-E_{\boldsymbol{\alpha}}(\mathrm{ref})=17.82718 \beta-17.69431 \beta=0.13287 \beta$ $\mathrm{BRE}($ monoradical $)=E_{\pi}-E_{\pi}(\mathrm{ref})=17.82718 \beta-17.69431 \beta=0.13287 \beta$ $\operatorname{BRE}($ monocation $)=E_{\pi}-E_{\pi}(\mathrm{ref})=17.82718 \beta-17.69431 \beta=0.13287 \beta$ $\mathrm{BRE}($ trication $)=E_{\pi}-E_{\pi}(\mathrm{ref})=15.82718 \beta-16.31561 \beta=-0.48843$

Figure 3. Bond resonance energy (BRE) of phenalenyl. 


\author{
(C) \\ hexagonal ring

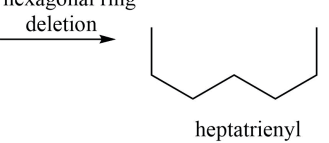 \\ $\mathrm{SC}=20$ \\ heptatrienyl \\ characteristic polynomial and eigenvalues \\ $\mathrm{X}^{13}-15 \mathrm{X}^{11}+84 \mathrm{X}^{9}-226 \mathrm{X}^{7}+309 \mathrm{X}^{5}-207 \mathrm{X}^{3}+54 \mathrm{X}=0$ \\ $\mathrm{X}=0, \pm 1, \pm 1, \pm 1, \pm 3^{1 / 2}, \pm 3^{1 / 2}, \pm 2.44949$ \\ trianion/diradical $E_{\pi}=15.82718 \beta$ \\ monoanion $E_{\pi}=17.82718 \beta$ \\ monoradical $E_{\pi}=17.82718 \beta$ \\ monocation $E_{\pi}=17.82718 \beta$ \\ trication/diradical $E_{\pi}=15.82718 \beta$ \\ $e f$ reference polynomial and eigenvalues \\ $P\left(G_{\text {ref }} ; \mathrm{X}\right)=P(G ; \mathrm{X})+2 P\left(G-r_{6} ; \mathrm{X}\right)$ \\ $\mathrm{X}^{13}-15 \mathrm{X}^{11}+84 \mathrm{X}^{9}-226 \mathrm{X}^{7}+309 \mathrm{X}^{5}-207 \mathrm{X}^{3}+54 \mathrm{X}$ \\ $\frac{2\left(X^{7}-6 X^{5}+10 X^{3}-4 X\right)}{X^{13}-15 X^{11}+84 X^{9}-224 X^{7}+297 X^{5}-187 X^{3}+46 X}=0$ \\ $\mathrm{X}^{13}-15 \mathrm{X}^{11}+84 \mathrm{X}^{9}-224 \mathrm{X}^{7}+297 \mathrm{X}^{5}-187 \mathrm{X}^{3}+46 \mathrm{X}=0$ \\ $\mathrm{X}=0, \pm 0.83584, \pm 0.83584, \pm 1.24777, \pm 1.76148, \pm 1.76148, \pm 2.428097$ \\ trianion/diradical $E_{\mathrm{T}}(\mathrm{ref})=16.06933 \beta$ \\ monoanion $E_{\pi}(\mathrm{ref})=17.74101 \beta$ \\ monoradical $E_{\pi}(\mathrm{ref})=17.74101 \beta$ \\ monocation $E_{\pi}(\mathrm{ref})=17.74101 \beta$ \\ trication/diradical $E_{\pi}(\mathrm{ref})=16.06933 \beta$ \\ ef(trianion $/$ diradical $)=E_{\pi}-E_{\pi}($ ref $)=15.82718 \beta-16.06933 \beta=-0.24215 \beta$ \\ ef(monoanion $)=E_{\pi}-E_{\pi}(\mathrm{ref})=17.82718 \beta-17.74101 \beta=0.08617 \beta$ \\ $e f($ monoradical $)=E_{\pi}-E_{\pi}(\mathrm{ref})=17.82718 \beta-17.74101 \beta=0.08617 \beta$ \\ ef(monocation $)=E_{\pi}-E_{\pi}(\mathrm{ref})=17.82718 \beta-17.74101 \beta=0.08617 \beta$ \\ ef $($ trication $/$ diradical $)=E_{\pi}-E_{\pi}(\mathrm{ref})=15.82718 \beta-16.06933 \beta=-0.24215$
}

Figure 4. Calculation of benzene hexagonal ring energy effect (ef) of phenalenyl.

where CCE is also called energy effect ef(G,Z) which is the effect of cycle $Z$ on the total $\pi$-energy of a conjugated molecule whose molecular graph is $G$. Using ef, it was shown that the empty hexagonal rings of total resonant sextet (TRS) benzenoids had a lower values [smaller CCE = ef(TRS, $\left.\mathrm{C}_{6}\right)$ values] compared to the Clar sextet rings. ${ }^{[4]}$

Removal of one of the equivalent benzene hexagonal rings from the phenalenyl molecular graph gives the heptatrienyl monoradical eliminating all the ciruits; ef = $0.08617 \beta$ for the any of the three equivalent peripheral hexagonal rings of phenalenyl (Figure 4) which is about one-third ( $32 \%$ ) the ef $=0.2726 \beta$ for benzene.

Using the three bond distinct ring lengths of 1.3942, 1.4199, and $1.4323 \AA$ computed via the B3L/YP/6-31+G* geometry optimization for the phenalenyl core, ${ }^{[17]}$ the harmonic oscillator model for ring aromaticity value was determined to be HOMA $=0.8704 .{ }^{[2]}$ For naphthalene HOMA $=0.811$ for each ring.

Global resonance energies (REs) of polycyclic conjugated hydrocarbons can be determined by conjugated circuit theory. ${ }^{[18]}$ Subsequently Randic has presented a way to partition global RE into resonance energy per each specific ring in any given benzenoid. ${ }^{[5]}$ This partitioning process gives values that measure the local ring

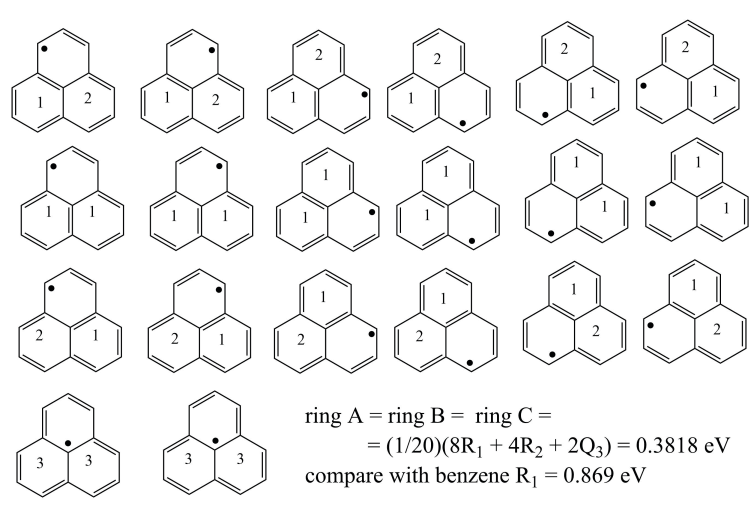

Figure 5. The 20 resonance structures of phenalenyl are listed above. Note that the six 2 nd row resonance structures with the number 1 enclosed within their rings are the more important resonance contributors with 2 C6 conjugated circuits and the lowest two resonance structures with the number 3 in their rings only have the C12 antiaromatic circuits and are the least important contributors.

aromaticity of benzenoids. We apply this method to the rings of phenalenyl. Figure 5 gives all the resonance structures $(S C=20)$ and summarizes our results. The resonance structures in Figure 5 have different Löwdin VB weights. ${ }^{[17,19]}$ Those resonance structures in Figure 5 having two rings enclosing the number 1 have Löwdin VB weights of $6.06 \%$, those having two rings enclosing the number 3 have Löwdin VB weights of $2.55 \%$, and the remaining ones have average Löwdin VB weights of $4.88 \%$. Thus, the resonance structures having the most benzene $\mathrm{C} 6$ circuits have a greater Löwdin VB weight and the ones without any have the lowest weight. The ring resonance energy for each phenalenyl ring is $\mathrm{RE} / \mathrm{ring}=0.382 \mathrm{eV}$ compared to benzene with $\mathrm{RE}=0.869 \mathrm{eV}$ and naphthalene with $0.652 \mathrm{eV}$. Of the 60 distinct rings in the 20 benzenoids listed in Figure 8 of Randić's paper 8 rings had lower values than 0.382/0.869 (normalized relative to benzene).[5] Overall phenalenyl rings have $44 \%$ the aromaticity of benzene.and are moderately aromatic by conjugated circuit theory.

\section{CONCLUSION}

The monocation, monoanion, and monoradical of phenalenyl are equally aromatic. Phenalenyl is moderately aromatic though kinetically reactive as determined by five different aromatic indices. Specifically, by BRE, ef, and partitioned conjugated circuit theory, the rings of phenalenyl have 49,32 , and $44 \%$ the aromaticity of benzene, respectively.

Acknowledgment. This work was supported in part by a grant from by the UM Board of Curators (K0906077). 


\section{REFERENCES}

[1] J. Aihara, Bull. Chem. Soc. Jpn 2016, 89, 1425-1454. https://doi.org/10.1246/bcsj.20160237

[2] T. M. Krygowski, M. K. Cyranski, Chem. Rev. 2001, 101, 1385-1419.

https://doi.org/10.1021/cr990326u

[3] S. Bosanac, I. Gutman, Z. Naturforsch. 1977, 32A, 10-12. https://doi.org/10.1515/zna-1977-0104

[4] I. Gutman, V. Ivanov-Petrovic, J. R. Dias, Polycycl. Aromat. Comp. 2000, 18, 221-229. https://doi.org/10.1080/10406630008028147

[5] M. Randić, J. Comput. Chem. 2019, 40, 753-762. https://doi.org/10.1002/jcc.25760

[6] M. Randic, Chem. Rev. 2003, 103, 3449-3605. https://doi.org/10.1021/cr9903656

[7] P. B. Sogo, M. J. Nakazaki, M. J. Calvin, J. Chem. Phys. 1957, 26, 1343. https://doi.org/10.1063/1.1743526

[8] K. Goto, T. Kubo, K. Yamamoto, K. Nakasuji, K. Sato, D. Shiomi, T. Takui, M. Kubota, T. Kobayashi, K. Yakusi, et al., J. Am. Chem. Soc. 1999, 121, 16191620. ttps://doi.org/10.1021/ja9836242

[9] S. Suzuki, Y. Morita, K.; Fukui, K. Sato, D. Shiomi, T. Takui, K. Nakasuji, J. Am. Chem. Soc. 2006, 128, 2530-2531. https://doi.org/10.1021/ja058387z
[10] M. K. Cyranski, R. W. A. Havenith, M. A. Dobrowolski, B. R. Gray, T. M. Krygowski, P. W. Fowler, L. W. Jenneskens, Chem. Eur. J. 2007, 13, 2201-2207. https://doi.org/10.1002/chem.200601619

[11] K. Balasubramanian, J. Comput. Chem. 1984, 5, 387394. https://doi.org/10.1002/jcc.540050417

[12] R. Ramaraj, K. Balasubramanian, J. Comput. Chem. 1985, 6, 122-141. https://doi.org/10.1002/jcc.540060207

[13] A. Das, T. Müller, F. Plasser, H. Lischka, J. Phys. Chem. A 2016, 120, 1625-1636. https://doi.org/10.1021/acs.jpca.5b12393

[14] J. R. Dias, Molec. Phys. 2013, 111, 735-751. https://doi.org/10.1080/00268976.2012.742584

[15] J. R. Dias, Molec. Phys. 2019, in press https://doi.org/10.1080/00268976.2019.1666172

[16] J. Aihara, Bull. Chem. Soc. Jpn 2007, 80, 1518-1521. https://doi.org/10.1246/bcsj.80.1518

[17] A. M. Toader, C. M. Buta, B. Frecus, A. Mischie, F. Cimpoesu, J. Phys. Chem. C 2019, 123, 6869-6880. https://doi.org/10.1021/acs.jpcc.8b12250

[18] M. A. Randić, Tetrahedron 1977, 33, 1905-1920. https://doi.org/10.1016/0040-4020(77)80375-X

[19] P. O. Löwdin, J. Molec. Struct. THEOCHEM 1991, 229, 114. https://doi.org/10.1016/0166-1280(91)90134-6 